\title{
Macrocytic anaemia status does not change in response to a physiological dose of folic acid in persons with suboptimal vitamin $B_{12}$ status
}

\author{
K. M. Mooney ${ }^{1}$, G. J. Cuskelly ${ }^{2}$ and I. Young ${ }^{3}$ \\ ${ }^{1}$ Liverpool Hope University, Hope Park, Liverpool L16 9JD, UK, ${ }^{2}$ Queen's University Belfast, Institute of Agri-Food and \\ Land Use, School of Biological Sciences, David Keir Bldg, Belfast BT9 5AG, UK and ${ }^{3}$ School of Medicine, Dentistry and \\ Biomedical Sciences, Queen's University Belfast, Belfast BT12 6BJ, UK
}

Mandatory folic acid fortification has been advocated as the most effective method of protecting against neural tube defect pregnancies on a population basis ${ }^{(1)}$. Most European countries have not proceeded due to concerns of putative masking of vitamin $B_{12}$ deficiency. However, it is currently unknown what dose of folic acid masks vitamin $\mathrm{B}_{12}$ deficiency.

The aim of this study was to assess the effect of a physiological dose of folic acid $(400 \mu \mathrm{g} / \mathrm{d})$ on the haematological profile of those with suboptimal vitamin $B_{12}$ status. Participants with serum vitamin $B_{12}$ levels between 130 and $200 \mathrm{ng} / \mathrm{l}$ were given either folic acid or placebo daily for 6 months in a randomized, double-blind, placebo-controlled study. Fasting blood samples were collected pre-intervention and at monthly intervals until completion to determine vitamin $\mathrm{B}_{12}$, folate and haematological status (mean cell volume, Hb and haematocrit).

Fourteen volunteers in the placebo group and 15 volunteers in the folic acid group completed the 6-month intervention. Volunteers who were Fe-deficient were excluded from analysis. There were no significant differences in vitamin B status and haematological markers between groups pre-intervention. Significant responses to intervention occurred in serum folate (increased; $P<0.001$ ), red blood cell folate (increased; $P=0.001$ ) and plasma homocysteine (decreased; $P=0.011$ ) in the folic acid group. Consumption of $400 \mu \mathrm{g}$ of folic acid per $\mathrm{d}$ had no significant effect on haematological profile. The table shows the numbers of volunteers who were defined as having a normal haematological status at each stage of intervention in each group.

\begin{tabular}{lccccccc}
\hline & \multicolumn{3}{c}{ Placebo } & & \multicolumn{3}{c}{ Folic acid } \\
\cline { 2 - 4 } \cline { 6 - 8 } & Pre-intervention & Post-intervention & $P$ & & Pre-intervention & Post-intervention & $P$ \\
\hline Mean cell volume & 10 & 10 & 9 & NS & 6 & 6 & NS \\
Hb & 9 & 8 & NS & 6 & 5 & NS \\
Haematocrit & 9 & 8 & 6 & 5 & NS \\
\hline
\end{tabular}

NS: non-significant. McNemar's test was used to determine differences in haematological status pre- and post-intervention within groups $(P<0.05)$.

This is the first folic acid supplementation trial in persons with apparent suboptimal vitamin $\mathrm{B}_{12}$ status. Both groups had a normal haematological profile pre-intervention suggesting that mean cell volume should not be the sole diagnostic marker used to identify possible vitamin $\mathrm{B}_{12}$ deficiency.

1. Oakley GP Jr (1997) Let's increase folic acid fortification and include vitamin B-12. Am J Clin Nutr 65, 1889-1890. 